\section{Taking a BET on hedgehog}

\section{By Kai-Jye Lou, Senior Writer}

Stanford University School of Medicine researchers have uncovered an interplay between BET bromodomain proteins and the hedgehog pathway that opens up additional oncology real estate for BET inhibitors. ${ }^{1}$ The inhibitors could be among the first molecules to work in hedgehog-driven cancers that no longer respond to smoothened inhibitors or other pathway modulators.

Ever since BET bromodomains emerged as therapeutic targets, corporate and academic groups have linked the epigenetic readers to long-standing cancer pathways such as c-Myc (MYC) and, more recently, androgen receptor signaling. ${ }^{2}$

Now, a research group led by Yoon-Jae Cho has published data in Nature Medicine suggesting that BET bromodomain inhibitors could also treat hedgehog-driven cancers that resist blockers of smoothened (SMO). Cho is an assistant professor in the departments of neurology and neurosurgery at the Stanford University School of Medicine.

SMO is a transmembrane signaling protein that sits near the top of the hedgehog pathway. It is perhaps the most tractable target in the pathway-there is one SMO inhibitor on the market and at least five others in the clinic-although mutations in SMO itself or downstream activation of the pathway can render such inhibitors ineffective. ${ }^{3-5}$

"We started by looking for ways to target as far downstream of smoothened as possible and began looking into the role of bromodomain proteins in regulating the transcriptional output of the hedgehog pathway," said Cho.

The BET bromodomain inhibitor JQ1-which is a research tool-disrupted hedgehog pathway signaling in mouse cell lines by decreasing transcription of glioma-associated oncogene homolog 1 zinc finger protein (Gli1) and Gli2, transcription

"We started by looking for ways
to target as far downstream of
smoothened as possible and
began looking into the role
of bromodomain proteins in
regulating the transcriptional
output of the hedgehog pathway."
$\quad-$ Yoon-Jae Cho,
Stanford University School of Medicine

resistant medulloblastoma. By contrast, vehicle and vismodegib did not.

Roche's Genentech Inc. unit markets Erivedge vismodegib to treat locally advanced and metastatic basal cell carcinoma (BCC). Novartis AG's sonidegib is under review in Europe to treat advanced BCC.

"The current study shows a way to potentially avoid classical resistance mechanisms that are somewhat contingent on direct inhibition of smoothened in the hedgehog pathway," said Arul Chinnaiyan. "From an academic perspective, this would be another lowhanging fruit for consideration with BET inhibitors. The efficacy results they've published thus far look very compelling."

Chinnaiyan is a professor of pathology and urology at the University of Michigan Medical School and an investigator at the Howard Hughes Medical Institute. He also is cofounder of OncoFusion Therapeutics Inc., which exclusively licensed a portfolio of BET bromodomain inhibitors to Medivation Inc. in April. ${ }^{6}$

\section{Safety first}

Cho said that BET inhibitors could be particularly useful for treating hedgehog-driven cancers with a priori resistance to SMO inhibitors as well as those that have acquired resistance following treatment. BCC and medulloblastomas are the two most common types of hedgehogdriven cancers, and the latter group has greater unmet needs, said Cho. "About $40 \%-50 \%$ of cases of hedgehog-driven medulloblastomas that come through the door are going to be resistant without having been treated with a smoothened inhibitor," he said.

Chinnaiyan thinks that BET inhibitors could benefit patients who are still sensitive to SMO inhibitors. "Depending on the toxicity profile, it could be possible to evaluate a BET inhibitor with a smoothened inhibitor to see if the combination would avoid the development of resistance," he said.

Because medulloblastomas are primarily factors that sit downstream of SMO. In hedgehog-driven mouse medulloblastoma cell lines, JQ1 inhibited growth with $\mathrm{IC}_{50}$ values of 50-150 nM.

JQ1 was more potent at decreasing viability and hedgehog pathway activity than vehicle or the SMO inhibitors vismodegib and sonidegib (LDE225) when tested in multiple hedgehog-driven, SMO inhibitorresistant mouse and human cancer cell lines. Also, JQ1 prevented tumor growth in a mouse allograft model of hedgehog-driven, SMO inhibitor- pediatric tumors, Cho expects that the safety bar for BET inhibitors will be higher than that for other types of cancer. However, the multiple pathways controlled by BET bromodomain proteins ${ }^{7}$ could also carry liabilities.

"The potential benefit of BET inhibitors is that they may be able to avoid proximal resistance mechanisms, such as mutations that occur in upstream targets of the hedgehog pathway like SMO and SUFU, but the trade-off could be increased toxicity," said Chinnaiyan. Suppressor of 


\section{ANALYSIS}

\section{TARGETS \& MECHANISMS}

fused homolog (SUFU) is a negative regulator of the hedgehog signaling that acts downstream of SMO.

Gerald Denis added that the bromodomain containing 4 (BRD4) protein co-represses multiple types of human integrated viruses and keeps them in a latent state, so inhibiting the protein's activity has the potential to reactivate such viruses and promote viremia. Denis is an associate professor of pharmacology and medicine at the Boston University School of Medicine.

Chinnaiyan is optimistic about the safety profile of BET inhibitors. "At least in early studies, JQ1 and other BET inhibitors have not shown marked toxicity when given at concentrations that kill the cancer cells," he told SciBX.

In April, Oncoethix S.A. reported interim Phase Ib data for its OTX015 small molecule BET bromodomain inhibitor that showed no dose-limiting toxicities in patients with acute leukemia and other hematologic malignancies.

Chinnaiyan said that it will be important to evaluate BET inhibition in additional xenograft models, especially in cases that are resistant to SMO inhibitors. He said that it also could be useful to investigate how BET inhibitors disengage the hedgehog pathway on a genomewide scale.

Cho said that his group is trying to identify synergistic combinations of BET inhibitors with inhibitors of SMO, histone deacetylases (HDACs) or the phosphoinositide 3-kinase (PI3K)/protein kinase B (PKB; PKBA; AKT; AKT1)/mammalian target of rapamycin (mTOR; FRAP; RAFT1) pathway. He said the group also is generating data that would be helpful for researchers who are planning clinical trials to evaluate BET inhibitors in pediatric cancers.

The results published in Nature Medicine are unpatented.

The Dana-Farber Cancer Institute has filed a patent application covering JQ1 and related analogs. The technology is licensed to Tensha Therapeutics Inc., which did not respond to requests for comment. Tensha has the JQ1-derived small molecule BET bromodomain inhibitor TEN-010 in Phase I testing to treat solid tumors.

Lou, K.-J. SciBX 7(29); doi:10.1038/scibx.2014.850

Published online July 31, 2014

\section{REFERENCES}

1. Tang, Y. et al. Nat. Med.; published online June 29, 2014; doi:10.1038/nm.3613

Contact: Yoon-Jae Cho, Stanford University School of Medicine, Stanford, Calif. e-mail: yjcho1@stanford.edu

2. Asangani, I.A. et al. Nature 510, 278-282 (2014)

3. Kim, J. et al. Cancer Cell 23, 23-34 (2013)

4. Buonamici, S. et al. Sci. Transl. Med. 2, 51ra70 (2010)

5. Atwood, S.X. et al. Nature 494, 484-488 (2013)

6. Lou, K.-J. SciBX 7(18); doi:10.1038/scibx.2014.510

7. Belkina, A.C. \& Denis, G.V. Nat. Rev. Cancer 12, 465-477 (2012)

\section{COMPANIES AND INSTITUTIONS MENTIONED}

Boston University School of Medicine, Boston, Mass. Dana-Farber Cancer Institute, Boston, Mass. Genentech Inc., South San Francisco, Calif. Howard Hughes Medical Institute, Chevy Chase, Md. Medivation Inc. (NASDAQ:MDVN), San Francisco, Calif. Novartis AG (NYSE:NVS; SIX:NOVN), Basel, Switzerland Oncoethix S.A., Lausanne, Switzerland OncoFusion Therapeutics Inc., Ann Arbor, Mich. Roche (SIX:ROG; OTCQX:RHHBY), Basel, Switzerland Stanford University School of Medicine, Stanford, Calif. Tensha Therapeutics Inc., Cambridge, Mass. University of Michigan Medical School, Ann Arbor, Mich. 